\title{
OPEN Author Correction: A modular cGAN classification framework: Application to colorectal tumor detection
}

\author{
Thomas E. Tavolara, M. Khalid Khan Niazi D, Vidya Arole, Wei Chen, Wendy Frankel \& \\ Metin N. Gurcan
}

Correction to: Scientific Reports https://doi.org/10.1038/s41598-019-55257-w, published online 12 December 2019

This Article contains errors in the Reference list. References 7, 10, 13, 16, 18, 19, 20, 21, 25, 27, 28, 30, 33, 35, 36 and 37 were incorrectly given as:

7. Niazi, M. K. K. et al. In Medical Imaging 2018: Digital Pathology. 105810 H (International Society for Optics and Photonics) (2018).

10. Niazi, M. K. K. et al. In Medical Imaging: Digital Pathology. 86760I (International Society for Optics and Photonics) (2013).

13. Japkowicz, N. In Proc. of the Int'l Conf. on Artificial Intelligence (2000).

16. Qin, Z., Zhang, C., Wang, T. \& Zhang, S. In International Conference on Advanced Data Mining and Applications. 1-11 (Springer) (2010).

18. Shaban, M. T., Baur, C., Navab, N. \& Albarqouni, S. J. a. p. a. StainGAN: Stain Style Transfer for Digital Histological Images (2018).

19. Bayramoglu, N., Kaakinen, M., Eklund, L. \& Heikkilä, J. In ICCV Workshops. 64-71 (2017).

20. Szegedy, C., Vanhoucke, V., Ioffe, S., Shlens, J. \& Wojna, Z. In Proceedings of the IEEE conference on computer vision and pattern recognition. 2818-2826 (2016).

21. Liu, Y. et al. Detecting Cancer Metastases on Gigapixel Pathology Images (2017).

25. Kohl, M., Walz, C., Ludwig, F., Braunewell, S. \& Baust, M. In International Conference Image Analysis and Recognition. 903-913 (Springer) (2018).

27. Tavolara, T. E. et al. Colorectal tumor identification by transferring knowledge from pan-cytokeratin to H\&E. Vol. 10956 MI (SPIE, 2019).

28. Niazi, M. K. K. et al. Generalization of tumor identification algorithms. Vol. 10956 MI (SPIE, 2019).

30. Wang, Z. J. h. e. u. c. z. w. r. s. The SSIM index for image quality assessment (2003).

33. Goodfellow, I. et al. In Advances in neural information processing systems. 2672-2680 (2016).

35. Isola, P., Zhu, J., Zhou, T. \& Efros, A. A. In 2017 IEEE Conference on Computer Vision and Pattern Recognition (CVPR). 5967-5976 (2017). 
36. Ronneberger, O. et al. U-Net Convolutional Networks for Biomedical Image Segmentation (2015).

37. Deng, J. et al. In Computer Vision and Pattern Recognition. CVPR 2009. IEEE Conference on. 248-255 (Ieee) (2009).

The correct references are listed below as refs. ${ }^{1-16}$.

\section{References}

1. Niazi, M. K. K. et al. Nuclear IHC enumeration: A digital phantom to evaluate the performance of automated algorithms in digital pathology. Medical Imaging 2018: Digital Pathology Vol. $10581105810 \mathrm{H}$ (International Society for Optics and Photonics, 2018).

2. Niazi, M. K. K. et al. Entropy based quantification of Ki-67 positive cell images and its evaluation by a reader study. Medical Imaging 2013: Digital Pathology. 86760I (International Society for Optics and Photonics, 2013).

3. Japkowicz, N. The class imbalance problem: Significance and strategies. Proc. of the Int'l Conf. on Artificial Intelligence (2000).

4. Qin, Z., Zhang, C., Wang, T. \& Zhang, S. Cost sensitive classification in data mining. International Conference on Advanced Data Mining and Applications. 1-11 (Springer, 2010).

5. Shaban, M. T., Baur, C., Navab, N. \& Albarqouni, S. StainGAN: Stain Style Transfer for Digital Histological Images. 2019 IEEE 16 th International Symposium on Biomedical Imaging (ISBI, 2019).

6. Bayramoglu, N., Kaakinen, M., Eklund, L. \& Heikkilä, J. Towards Virtual H\&E Staining of Hyperspectral Lung Histology Images Using Conditional Generative Adversarial Networks. ICCV Workshops. 64-71 (2017).

7. Szegedy, C., Vanhoucke, V., Ioffe, S., Shlens, J. \& Wojna, Z. Rethinking the inception architecture for computer vision. Proceedings of the IEEE conference on computer vision and pattern recognition. 2818-2826 (2016).

8. Liu, Y. et al. Detecting Cancer Metastases on Gigapixel Pathology Images. arXiv preprint arXiv:1703.02442. (2017).

9. Kohl, M., Walz, C., Ludwig, F., Braunewell, S. \& Baust, M. Assessment of Breast Cancer Histology Using Densely Connected Convolutional Networks. International Conference Image Analysis and Recognition. 903-913 (Springer, 2018).

10. Tavolara, T. E. et al. Colorectal tumor identification by transferring knowledge from pan-cytokeratin to H\&E. Medical Imaging 2019: Digital Pathology. Vol. 10957 MI. (SPIE, 2019).

11. Niazi, M. K. K. et al. Generalization of tumor identification algorithms. Medical Imaging 2019: Digital Pathology. Vol. 10956 MI (SPIE, 2019).

12. Wang, Z. The SSIM index for image quality assessment. (2003).

13. Goodfellow, I. et al. Generative adversarial nets. Advances in neural information processing systems. 2672-2680 (2014).

14. Isola, P., Zhu, J., Zhou, T. \& Efros, A. A. Image-to-Image Translation with Conditional Adversarial Networks. 2017 IEEE Conference on Computer Vision and Pattern Recognition (CVPR). 5967-5976 (2017).

15. Ronneberger, O. et al. U-Net Convolutional Networks for Biomedical Image Segmentation. International Conference on Medical image computing and computer-assisted intervention, 234-241 (2015).

16. Deng, J. et al. Imagenet: A large-scale hierarchical image database. Computer Vision and Pattern Recognition, 2009. CVPR 2009. IEEE Conference on. 248-255 (Ieee, 2009).

(c) (i) Open Access This article is licensed under a Creative Commons Attribution 4.0 International License, which permits use, sharing, adaptation, distribution and reproduction in any medium or format, as long as you give appropriate credit to the original author(s) and the source, provide a link to the Creative Commons license, and indicate if changes were made. The images or other third party material in this article are included in the article's Creative Commons license, unless indicated otherwise in a credit line to the material. If material is not included in the article's Creative Commons license and your intended use is not permitted by statutory regulation or exceeds the permitted use, you will need to obtain permission directly from the copyright holder. To view a copy of this license, visit http://creativecommons.org/licenses/by/4.0/.

(C) The Author(s) 2020 